\title{
ASPECTOS MICROBIOLÓGICOS DE CENOURAS MINIMAMENTE PROCESSADAS E ARMAZENADAS EM DIFERENTES EMBALAGENS SOB-REFRIGERAÇÃO
}

\section{MICROBIOLOGICAL ASPECTS OF CARRIES MINIMALLY PROCESSED AND STORED IN DIFFERENT UNDER-COOLING PACKS}

\author{
L. P. PASSOS ${ }^{1}$, A. L. S. MIRANDA ${ }^{1}$, D. R. P. MARQUES ${ }^{1}$, I. R. N. OLIVEIRA ${ }^{1}$ \\ ${ }^{1}$ Instituto de Ciências Agrárias. Universidade Federal de Viçosa - Campus Rio Paranaíba \\ E-mail: E-mail: louise.passos@ufv.br
}

\author{
article info \\ Article history: \\ Received 12 May 2017 \\ Accepted 3 August 2017 \\ Available online 20 September 2017
}

PALAVRAS-CHAVE: Baby carrot; Cenourete; Daucus carota L; Coliformes totais.

KEYWORDS: Baby carrot; Cenourete; Daucus carota L; Total coliforms.

\begin{abstract}
RESUMO: Frutas e hortaliças minimamente processadas têm mostrado crescimento expressivo nos últimos anos, em virtude de grandes mudanças no estilo de vida do consumidor, como a conscientização da necessidade de uma dieta alimentar saudável e que cumpra as exigências de segurança alimentar. A cenoura é uma das mais populares, pela sua versatilidade de uso e formas de apresentação ao consumidor. Este trabalho teve como objetivo avaliar a eficiência da etapa de sanitização e o aspecto microbiológico de cenouras minimamente processadas armazenadas em diferentes embalagens. As hortaliças foram minimamente processadas e avaliadas microbiologicamente quanto ao teor de coliformes a $35^{\circ} \mathrm{C}$, coliformes a $45^{\circ} \mathrm{C}$ e psicrotróficos. Observou-se que a sanitização da hortaliça foi eficiente, visto que a contagem microbiana para coliformes teve diminuição após o processamento. A embalagem não influenciou no crescimento de microrganismos. A contagem de coliformes estavam dentro dos limites estabelecidos pela legislação. O produto foi considerado seguro até o $7^{\circ}$ dia de armazenamento, após esse período a contagem de psicrotróficos foi elevada.
\end{abstract}

\begin{abstract}
Minimally processed fruits and vegetables have shown significant growth in recent years due to major changes in the consumer's lifestyle, such as awareness of the need for a healthy diet that meets food safety requirements. The carrot is one of the most popular, for its versatility of use and forms of presentation to the consumer. This work aimed to evaluate the efficiency of the sanitization step and the microbiological aspect of minimally processed carrots stored in different packages. The vegetables were minimally processed and evaluated microbiologically for coliform content at $35{ }^{\circ} \mathrm{C}$, coliforms at $45{ }^{\circ} \mathrm{C}$ and psychrotrophic. It was observed that the sanitation of the vegetables was efficient, since the microbial count for coliforms decreased after processing. The packaging did not influence the growth of microorganisms. The coliform count was within the limits set by legislation. The product was considered safe until the 7th day of storage, after that period the psychrotrophic count was elevated.
\end{abstract}

\section{INTRODUÇÃO}

Das hortaliças minimamente processadas, a cenoura (Daucus carota L.) é uma das mais populares, pela sua versatilidade de uso e formas de apresentação ao consumidor, podendo ser 


\section{The Journal of Engineering and Exact Sciences - \\ JCEC \\ ISSN: 2527-1075}

ralada em diversos tamanhos ou cortada na forma de fatias, cubos, palitos, e ainda apresentada na forma de mini-cenoura baby-carrot.

As deformações na cenoura podem chegar a 30\% da produção (SABIO et al., 2015), o que acarreta em perdas econômicas, e uma alternativa para agregar valor seria o beneficiamento deste produto, como a produção de produtos minimamente processados.

Hortaliças e frutas minimamente processadas podem ser definidas como produtos que, embora fisicamente alterados, permanecem em estado fresco e, na maioria das vezes, não necessitam de preparo subsequente para consumo (SANTOS et al., 2010). O processamento mínimo inclui as etapas de seleção/classificação da matéria prima, pré-lavagem, processamento (corte, fatiamento), santificação, enxágue, centrifugação e embalagem (SANTOS et al., 2010) que interferem nos fatores físicos, químicos e biológicos responsáveis pela deterioração do produto. Por exemplo, cortes ou danos no tecido da planta promovem liberação de nutrientes e enzimas intracelulares que favorecem a atividade enzimática e a proliferação de microrganismos (FANTUZI, PUSCHMANN e VANETTI, 2004).

Devido à elevada manipulação e ao incremento no consumo de minimamente processados, tanto em âmbito doméstico quanto institucional, a preocupação com o risco potencial para a saúde pública aumentou, devido à probabilidade de contaminação microbiológica (SANTOS et al., 2010). Os minimamente processados têm também sido veículos de contaminação por bactérias envolvidas em surtos, que constituem um grande problema de saúde pública, sendo responsáveis por elevados custos econômicos (ASSIS, 2014).

Diante do exposto, o objetivo do presente trabalho foi avaliar a eficiência da etapa de sanitização e o aspecto microbiológico de cenouras minimamente processadas armazenadas em diferentes embalagens.

\section{MATERIAL E MÉTODOS}

As cenouras foram adquiridas no comércio de Rio Paranaíba-MG e avaliadas quanto as características microbiológicas antes do processamento para verificar a qualidade da matériaprima. Em seguida, as cenouras foram processadas no Centro Vocacional Tecnológico (CVT) de Rio Paranaíba. Inicialmente foram lavadas e sanitizadas (hipoclorito de sódio a $100 \mathrm{ppm} /$ 15 min), sendo enxaguadas com água corrente e em solução de hipoclorito de sódio a 5 ppm. As cenouras cortadas e descascadas foram torneadas em boleador mecânico, obtendo cenouretes e catetinhos (as mini-cenouras brasileiras). Após o boleamento os produtos foram enxaguados com solução de hipoclorito de sódio a $20 \mathrm{ppm}$. Antes do envase as amostras foram novamente submetidas a caracterização microbiológica para verificar a eficiência da sanitização e verificação das Boas Práticas de Fabricação.

As amostras foram acondicionadas em bandeja de poliestireno (OS) com filme de policloreto de polivinila (PVC) e em potes de polipropeno (PP), e encaminhadas para armazenamento refrigerado $\left(7^{\circ} \mathrm{C}\right)$ durante 8 dias, sendo realizada análises periódicas. 


\section{The Journal of Engineering and Exact Sciences - \\ JCEC \\ ISSN: 2527-1075}

Os produtos foram analisados quanto as concentrações de psicrotróficos e coliformes a $35{ }^{\circ} \mathrm{C}$ e $45^{\circ} \mathrm{C}$ (APHA, 2001). As análises foram realizadas no Laboratório de Microbiologia de Alimentos da Universidade Federal de Viçosa- Campus de Rio Paranaíba.

O experimento foi conduzido em delineamento inteiramente casualizado (DIC) em arranjo fatorial, com dois fatores: embalagem $\mathrm{X}$ tempo. Os resultados foram avaliados por meio de análise de variância (ANOVA) e regressão. Os modelos foram escolhidos com base na significância dos coeficientes de regressão (teste t, ao nível de 5\% de probabilidade). Quando a regressão não foi significativa, os dados foram avaliados por meio do teste Tukey, ao nível de 5\% de significância. Todas as análises estatísticas foram conduzidas utilizando-se o programa estatístico R (The R Project for Statistical Computing), versão 3.3.0(2016).

\section{RESULTADOS E DISCUSSÃO}

A qualidade microbiológica da matéria-prima é muito importante na elaboração de frutas e hortaliças minimamente processadas, sendo um parâmetro importante na escolha dos fornecedores e procedência da matéria-prima. Isto é determinante, pois os produtos minimamente processados não possuem etapas que garantem a eliminação total de agentes microbiológicos. A etapa de sanitização empregada não possui completa eficiência se a hortaliça já vier com uma contaminação elevada. Sendo assim, uma carga microbiana inicial baixa resultará em um produto de melhor qualidade (CENCI, 2011).

A contagem microbiana inicial da matéria-prima utilizada está descrita na Tabela 1.

Tabela 1 - Caracterização microbiológica das amostras de cenouras antes (in natura) e após o processamento

Parâmetros Microbiológicos In natura Minimamente processado

\begin{tabular}{ccc}
\hline Coliformes a $35^{\circ} \mathrm{C}(\log \mathrm{NMP} / \mathrm{g})$ & $1,04 \pm 0,0 \mathrm{a}$ & $0,53 \pm 0,08 \mathrm{~b}$ \\
Coliformes a $45^{\circ} \mathrm{C}(\mathrm{Log} \mathrm{NMP} / \mathrm{g})$ & $1,04 \pm 0,0 \mathrm{a}$ & $0,47 \pm 0,0 \mathrm{~b}$ \\
Psicrotróficos (Log UFC/g) & $0,0 \pm 0,0$ & $0,0 \pm 0,0$
\end{tabular}

Médias seguidas de letras diferentes, na linha, diferem estatisticamente pelo teste Tukey $(\alpha=0,05)$.

Dentre os parâmetros microbiológicos analisados, as amostras in natura e minimamente processadas são estatisticamente diferentes pelo teste Tukey, mostrando que as cenouras in natura apresentavam uma contagem microbiana mais elevada quando comparadas as cenouras minimamente processadas, que passaram por etapas de sanitização. Esse resultado comprova a eficiência do processo quanto à redução de contagem dos microrganismos analisados, estando dentro do limite exigido pela legislação brasileira (RDC n 12/2001) (Brasil, 2001), e comprova o cumprimento das Boas Práticas de Fabricação.

Segundo Moretti (2007), cada estágio do fluxograma do processamento mínimo de hortaliças requer a aplicação de práticas higiênico-sanitárias eficazes, para garantir que os perigos sejam controlados e que se obtenha um produto de qualidade e seguro. 


\section{The Journal of Engineering and Exact Sciences - \\ JCEC \\ ISSN: 2527-1075}

Para hortaliças in natura e preparadas, a resolução $\operatorname{RDC} n^{\circ} 12$, de 02 de janeiro de 2001 regulamenta sobre padrões microbiológicos e define como limite de coliformes a $45{ }^{\circ} \mathrm{C}$ um teor de $10^{2} \mathrm{NMP} / \mathrm{g}$. Analisando os resultados da Tabela 2, nota-se que as amostras após 8 dias de armazenagem encontram-se dentro deste limite estabelecido para coliformes a $45^{\circ} \mathrm{C}$.

Tabela 2 - Contagem microbiológica de coliformes a $35^{\circ} \mathrm{C}$ e $45^{\circ} \mathrm{C}$ em cenoura armazenada sob condições de refrigeração $\left(7^{\circ} \mathrm{C}\right)$ em pote de polipropeno (PP) e filme de PVC

\begin{tabular}{|c|c|c|c|c|}
\hline \multirow{2}{*}{ Tempos (dias) } & \multicolumn{2}{|c|}{ Coliformes a $35^{\circ} \mathrm{C}(\mathrm{NMP} / \mathrm{g})$} & \multicolumn{2}{|c|}{ Coliformes a $45^{\circ} \mathrm{C}(\mathrm{NMP} / \mathrm{g})$} \\
\hline & Pote PP & PVC & Pote PP & $\overline{\text { PVC }}$ \\
\hline 0 & $3,4 \pm 0,26^{\mathrm{ns}}$ & $3,4 \pm 0,26^{\mathrm{ns}}$ & $3,0 \pm 0,0^{\mathrm{ns}}$ & $3,0 \pm 0,0^{\mathrm{ns}}$ \\
\hline 4 & $4,63 \pm 1,77^{\text {ns }}$ & $3,4 \pm 0,26^{\mathrm{ns}}$ & $3,0 \pm 0,0^{\text {ns }}$ & $3,0 \pm 0,0^{\mathrm{ns}}$ \\
\hline 8 & $3,2 \pm 0,26^{\mathrm{ns}}$ & $5,6 \pm 3,55^{\mathrm{ns}}$ & $3,0 \pm 0,0$ ns & $3,0 \pm 0,0 \mathrm{~ns}$ \\
\hline
\end{tabular}

$\mathrm{ns}=$ Médias no mesmo grupo de microrganismo, na linha e coluna, são iguais pelo teste Tukey $(\alpha=0,05)$.

A legislação, RDC n ${ }^{\circ} 12 / 2001$, não descreve valores para coliformes a $35{ }^{\circ} \mathrm{C}$, mas segundo Berbari, Paschoalino \& Silveira (2001) e Smaniotto et al. (2009) contagem de coliformes totais $\geq 1.100 \mathrm{NMP} / \mathrm{g}$ são consideradas elevadas e indica a falta ou falha do cumprimento das Boas Práticas de Fabricação, sendo as condições higiênico-sanitárias insatisfatórias no processamento ou pós-processamento (armazenagem). Considerando esses limites, as cenouras apresentavam-se com contagem de coliformes adequadas até o $8^{\circ}$ dia de armazenamento, evidenciado as boas condições sanitárias durante o processamento e armazenamento refrigerado $\left(7^{\circ} \mathrm{C}\right)$.

Observou-se pela ANOVA que não houve interação significativa ( $p>0,05)$ entre tempo e embalagem para a concentração de psicrotróficos. A análise estatística mostrou que a embalagem não apresentou efeito sobre o crescimento $(\mathrm{p}>0,05)$ deste grupo de microrganismos, fato que pode ser observado na Figura 1, onde as curvas de desenvolvimento dos microrganismos psicrotróficos para as duas embalagens estão próximas.

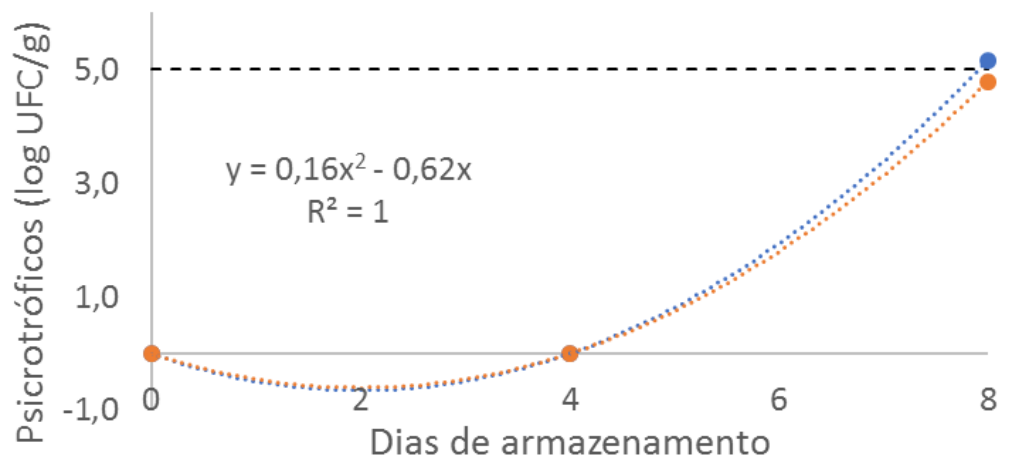

Figura 1 - Contagem microbiológica de psicrotróficos em cenoura minimamente processada armazenada em pote de polipropeno (PP) (•) e bandejas com filme de PVC (॰). (---) Limite recomendado por VITTI et.al (2004).

O tempo teve influência significativa $(\mathrm{p}<0,05)$ na contagem de psicrotróficos, apresentando variação ao longo da armazenagem. A legislação não relata limites para esse grupo de microrganismo, porém há uma recomendação que alimentos com contagens de 


\section{The Journal of Engineering and Exact Sciences - \\ JCEC}

ISSN: 2527-1075

psicrotróficos superior a $105 \mathrm{UFC/g}(5 \mathrm{log} \mathrm{UFC} / \mathrm{g}$ ) podem ser considerados impróprios para consumo humano devido a perda do valor nutricional, alterações sensoriais e riscos de contaminação (VITTI et. al, 2004).

O modelo de $2^{\mathrm{a}}$ ordem se ajustou a variação do crescimento de psicrotróficos, podendo a curva ser dividida em dois estágios: Fase lag de desenvolvimento até o $4^{\circ}$ dia de armazenamento, apresentando pouco desenvolvimento; seguido de uma fase exponencial de crescimento (característica da fase $\log$ ). Segundo a equação, as amostras estariam aptas para o consumo até o $7^{\circ}$ dia de armazenamento, apresentando 3,5 $\log \mathrm{UFC} / \mathrm{g}$, limite inferior ao proposto por VITTI et. al. (2004). A partir do $8^{\circ}$ dia de estocagem a contagem de psicrotróficos foi elevada (> $5 \mathrm{log}$ UFC/g) e não é possível garantir a segurança do produto. Este limite é considerado perigoso não apenas por acelerar o processo de deterioração, mas por indicar riscos da presença de bactérias patogênicas frio-tolerantes (ROSA et.al, 2001).

\section{CONCLUSÕES}

A sanitização mostrou-se eficiente para reduzir a contagem microbiológica inicial. $\mathrm{O}$ tipo de embalagem não interferiu no desenvolvimento de coliformes (totais e termotolerantes) e microrganismos psicrotróficos. A contagem de coliformes estava dentro dos limites estabelecidos pela legislação. As cenouras minimamente processadas apresentaram características microbiológicas satisfatórias até do $7^{\circ}$ dia de armazenagem, onde a vida útil do produto foi limitada pelo desenvolvimento elevado de psicrotróficos a partir do $8^{\circ}$ dia de armazenamento refrigerado $\left(7^{\circ} \mathrm{C}\right)$.

\section{REFERÊNCIAS}

APHA. Compendium of the methods for the microbiological examination of foods. 4.ed. Washington: 2001. 676p.

BRASIL. Agência Nacional de Vigilância Sanitária. Resolução $\operatorname{RDC}^{0} \mathbf{1 2}$, de 02 de janeiro de 2001. Aprova o regulamento técnico sobre padrões microbiológicos para alimentos. Diário Oficial da União de 10 de janeiro de 2001, DOU, Seção 1, n 7-Em p. 45-53, Brasília, DF, 2001.

ASSIS, L.L.R.; UCHIDA, N.S. Análise da qualidade microbiológica de hortaliças minimamente processadas comercializadas em Campo Mourão, PR. Brazilian Journal Surgery Clinical Research, v.5, n.3, p.17-22, 2014.

BERBARI, S.A.G.; PASCHOALINO, J.E.; SILVEIRA, N.F.A. Efeito do cloro na água de lavagem para desinfecção de alface minimamente processada. Ciência e Tecnologia de Alimentos, v.21, n.2, p.1-5, 2001.

CENCI, S. A. Processamento mínimo de frutas e hortaliças. Tecnologia, qualidade e sistemas de embalagem. 1a edição.ed. Rio de Janeiro: Embrapa Agroindústria de Alimentos, 2011. 143p.

MORETTI, C.L. Panorama do processamento mínimo de frutas e hortaliças. Manual de Processamento Mínimo de Frutas e Hortaliças. Brasília, cap. 01, p.25-39, 2007.

ROSA, O. O.; CARVALHO, E. P.; DÍONIZIO, F.L.; RIBEIRO, A.C.; BEERLI, K.M. Indicadores de contaminação ambiental e condições higiênicas insatisfatórias de processamento em hortaliças minimamente processadas. Higiene Alimentar, v.18, n.122, p.74-84, 2004. 
SABIO, R. P.; GARCIA, J. B.; DUARTE, E. N.; PACHECO, A. L. A. A vez dos feios. HF Brasil. Hortifruti/Cepea, 2015. p. 10-18.

SMANIOTO, T.F.; PIROLO, N.J.; SIMIONATO, E.M.R.S.; ARRUDA, M.D. de. Contribuição ao estudo da qualidade microbiológica de frutas e hortaliças minimamente processadas. Revista Instituto Adolfo Lutz, v.68, n.1 p.150, 2009.

SANTOS, T.B.A.; SILVA, N. da; JUNQUEIRA, V. C. A.; PEREIRA, J. L. Microrganismos indicadores em frutas e hortaliças minimamente processadas. Revista Brazilian Journal Food Technology. v.13, n.2, p.141-146, 2010.

VITTI, M.C.D.; KLUGE, R.A.; GALLO, C.R.; SCHIAVINATO, M.A.; MORETTI, C.L.; JACOMINO, A.P. Aspectos fisiológicos e microbiológicos de beterrabas minimamente processadas. Pesquisa Agropecuária Brasileira, v.39, n.10, p. 1027-1032, 2004. 